\title{
Tendinopathien der unteren Extremität im Sport - Diagnostik und Therapie
}

\author{
Michael Cassel, Josefine Stoll, Frank Mayer
}

\section{Zusammenfassung}

Tendinopathien sind häufig Ursache chronischer, belastungsabhängiger Beschwerden an der unteren Extremität. Zumeist sind die großen Sehnen an Sprung- und Kniegelenk, insbesondere die Achillessehne und die Patellarsehne, betroffen. Ätiologisch wird eine repetitive Überbeanspruchung in Sport und/oder Alltag angenommen. Neben der klinischen Untersuchung und der ausführlichen Schmerz- und Belastungsanamnese hat die Sonografie als bildgebendes Verfahren einen hohen
Stellenwert in der Diagnostik von Sehnenpathologien der unteren Extremität. Als Mittel der Wahl in der Behandlung sind trainingstherapeutische Konzepte anzusehen, die mit physiotherapeutischen Maßnahmen kombiniert werden können. Bei höhergradiger Pathologie und länger bestehenden Beschwerden sollten multimodale Therapieansätze genutzt werden. Erst bei Versagen der konservativen Behandlung über zumindest mehrere Monate sollte der Einsatz eines Operationsverfahrens erwogen werden.

\section{Einleitung}

Sehnenpathologien sind häufig Ursache chronischer, belastungsabhängiger Beschwerden an der unteren Extremität. Je nach Ausprägungsgrad resultiert nicht selten eine deutliche Einschränkung der sportlichen und/oder alltäglichen Belastbarkeit. In der Mehrzahl der Fälle berichten die Patienten bereits bei Erstvorstellung über eine längere Beschwerdedauer, charakteristischerweise mit Anlaufschmerzen am Morgen, zu Beginn von Belastungen und bei Zunahme der Belastungsintensität. Zumeist sind die großen Sehnen an Sprung- und Kniegelenk, insbesondere die Achillessehne und die Patellarsehne, betroffen. Weniger häufig finden sich Pathologien der Quadrizepssehne, des Tractus iliotibialis, der Sehne des M. biceps femoris, des M. tibialis posterior sowie der Ursprünge der hüftumgreifenden Muskulatur (v.a. Sehnen

Dieser Artikel wurde erstmalig publiziert in Orthopädie und Unfallchirurgie up2date 2012; 7: 167-183 und liegt hier in aktualisierter Form von 2015 vor.

OP-JOURNAL 2016; 32: 44-54

(c) Georg Thieme Verlag KG Stuttgart · New York DOI http://dx.doi.org/10.1055/s-0042-111250

des M. rectus femoris und der Adduktorenmuskulatur).

Ätiologisch wird in der Regel eine repetitive Überbeanspruchung in Sport und/ oder Alltag genannt, wobei unterschiedliche Erklärungsmodelle diskutiert werden. So wird angenommen, dass eine hohe Beanspruchung zu Mikrorupturen im Kollagengewebe führt, die regenerative Prozesse nach sich ziehen. Hierbei findet u.a. ein struktureller Umbau mit vermehrtem Aufbau von Kollagen Typ III statt. Zur Schmerzgenese werden derzeit bevorzugt die Einsprossung von Mikrogefäßen und freien Nervenendigungen, die Hochregulierung der schmerzauslösenden Substanz P bei Hyperzellularität und Gefäßproliferation sowie eine mechanische Kompression durch benachbarte Strukturen diskutiert. Die zugrunde liegenden Mechanismen der Modelle sind jedoch bislang nicht abschließend geklärt.

In der klinischen Untersuchung zeigt sich die betroffene Sehne lokal druckdolent und in der subakuten und chronischen Phase ohne klinische Zeichen einer Entzündung wie Schwellung, Rötung oder Überwärmung. Je nach Stadium ist eine lokale Auftreibung bzw. Knötchenbil- dung in der Sehnenstruktur sichtbar bzw. tastbar. Die bildgebende Diagnostik beschränkt sich zunächst auf die Sonografie. Hierin sind Sehnendurchmesser, hypoechogene und hyperechogene Areale und Zonen als Indikator für intratendinöse Läsionen verlässlich $\mathrm{zu}$ erfassen und ggf. zu quantifizieren. Zudem sind diese im Krankheitsverlauf ohne größeren Zeit- und Kostenaufwand einfach und schnell kontrollierbar. Doppler-sonografisch sichtbare intratendinöse Gefäße sind als Zeichen des Sehnenumbaus von prognostischer und diagnostischer Bedeutung.

In der Therapie wird derzeit diskutiert, ob aktuelle Maßnahmen tatsächlich die pathophysiologische Basis berücksichtigen oder ob nicht nach wie vor eine Behandlung der Symptome im Vordergrund steht. Für Achilles- und Patellarsehnentendinopathien ist die Behandlung mittels exzentrischen Trainings evident. Weiterhin zeigte die extrakorporale Stoßwellentherapie besonders bei Ansatztendinopathien hohe Wirksamkeit. Der Nachweis in der Praxis häufig angewandter physiotherapeutischer und physikalischer Maßnahmen (u.a. Querfriktionen, therapeutischer Ultraschall) oder lokaler Infiltrationen (u.a. Steroide, Platelet-Rich-Plasma, sklerosierende Substanzen) wurde bislang hingegen nicht abschließend geführt. Ein belegter Nachteil der Kortisoninfiltration liegt in der möglichen, nachfolgenden Gewebedegeneration mit erhöhter Rupturgefahr, insbesondere bei sportlich ambitionierten Patienten und Athleten.

\section{Epidemiologie}

Epidemiologische Daten der letzten Jahre zeigen eine Zunahme chronischer Sehnenbeschwerden sowohl bei Athleten unterschiedlicher Sportarten als auch bei Personen mit körperlicher Belastung am Arbeitsplatz. In erster Linie resultiert eine Einschränkung der sport- 
lichen Belastbarkeit bzw. der Arbeitsfähigkeit.

Verletzungen und Überlastungsbeschwerden an Sehnen betreffen bis zu 50\% der behandlungsbedürftigen Diagnosen im Sport. Wissenschaftliche Untersuchungen zum Symptomenkomplex der Tendinopathien wurden vorwiegend an Achilles- und Patellarsehnen durchgeführt, die je nach Belastungsprofil die häufigsten Lokalisationen von Sehnenpathologien darstellen. In einer belgischen Querschnittsstudie an einer großen Kohorte fand sich kein Geschlechterunterschied [22]. Bei Laufsportlern sind am häufigsten Tendinopathien der Achillessehnen zu finden. Weitere Lokalisationen sind die Patellarsehne und der Tractus iliotibialis. Seltener, jedoch zumeist von langer Dauer, ist die Plantarfaszie betroffen. Ergebnisse einer retrospektiven Befragung von Nachwuchsläufern (13-18 Jahre) ergaben eine ähnliche Verteilung von Beschwerden an Achillessehne (6-9\%), Tractus iliotibialis (5-7\%) und Plantarfaszie (3-5\%). Ein vorderer Knieschmerz bestand bei diesem Kollektiv bei etwa jedem fünften Sportler, der Anteil der Patellarsehnenbeschwerden wurde dabei nicht differenziert angegeben [16]. In einer aktuellen QuerschnittsUntersuchung an 760 Nachwuchsathleten (13 \pm 2 Jahre; Spanne 8-18 Jahre) fand sich eine Achillessehnen-Tendinopathie bei $1,8 \%$ und eine PatellasehnenTendinopathie bereits bei 5,6\% der Sportler [17]. Bei erwachsenen Laufsportlern im Breiten- und Spitzensport wird, je nach Autor, über Sehnenbeschwerden in einer Häufigkeit zwischen 7 und 30\% berichtet [22,23]. Demgegenüber stehen in Spiel- und Sprungsportarten Beschwerden der Patellarsehne, meist als „Jumper's Knee“ bezeichnet, im Vordergrund. Bei Fußballern sind zudem gehäuft Tendinopathien der Adduktorenmuskulatur zu finden. Die Inzidenz von Beschwerden und Verletzungen der Hüftregion wird bei Fußballern mit 10 18 pro 100 Spieler im Jahr angegeben, genauere Angaben zu Beschwerden und Pathologien einzelner Sehnen liegen allerdings nicht vor [24].

\section{Risikofaktoren zur Entwicklung einer Tendinopathie}

Zu den Risikofaktoren in der Genese von Tendinopathien existieren derzeit nur wenige Daten aus wissenschaftlichen Längsschnittuntersuchungen. Neben einer sportartspezifischen Häufung (Achillessehnenbeschwerden bei Läufern, Pa- tellarsehnenbeschwerden bei Athleten aus Sprungsportarten) werden vor allem Vorverletzungen als wichtiger Risikofaktor angesehen. In einer aktuellen Literaturübersicht über Risikofaktoren zur Entwicklung einer Patellarsehnentendinopathie wurden 8 mögliche Einflussfaktoren genannt:

- das Gewicht,

- der BMI,

- die sog. „Waist-to-Hip-Ratio“,

- eine bestehende Beinlängendifferenz,

- ein erniedrigtes Fußlängsgewölbe,

- die Flexibilität der Quadrizeps- und ischiokruralen Muskulatur,

- die Kraft der Quadrizepsmuskulatur,

- die vertikale Sprungkraft [18].

Aufgrund der zumeist nicht ausreichenden Evidenz sind diese Faktoren bzw. deren Aussagekraft nach Meinung der Autoren nur mit Einschränkung zu werten. Folglich findet die Einteilung der Risikofaktoren meist auf klinischer Basis in extrinsische und intrinsische Faktoren statt. Eine Wertung möglicher Prädiktoren ist dabei allerdings nur begrenzt möglich (Tab.1). Extrinsische Faktoren umfassen die äußeren Einflüsse wie Trainingsfehler (zu hohe Trainingsumfänge und -intensitäten, ungünstige Geländewahl), Umgebungsbedingungen und Ausrüstung. Intrinsische Faktoren beziehen sich überwiegend auf die individuellen Voraussetzungen sowie den Trainingszustand (Tab. 1). Als valide intrinsische Risikofaktoren in der Entwicklung einer Achillessehnentendinopathie konnten neben dem Alter das Körpergewicht und die Körpergröße belegt werden [25].

Risikofaktoren zur Entwicklung einer Tendinopathie werden in der Regel in extrinsische und intrinsische Faktoren unterteilt. In erster Linie sind dabei Trainingsfehler, Ausrüstung sowie individuelle biomechanische Voraussetzungen und der Trainingszustand zu nennen. Wissenschaftliche Längsschnittuntersuchungen zum evidenzbasierten Beleg stehen jedoch noch aus.

\section{Die Tendinopathie in Abgrenzung zu Tendinose und Tendinitis}

Mit dem Begriff der Tendinopathie werden schmerzhafte Prozesse an Sehnen umschrieben. Inwieweit bereits strukturelle Veränderungen vorliegen, wird durch diesen Terminus allerdings nicht definiert. In der Mehrzahl der Fälle fehlen klinische und histologische Entzündungszeichen. Entgegen früherer An- nahmen konnten in Mikrodialysen keine Entzündungszellen und -mediatoren in den betroffenen Sehnen gefunden werden. In akuten Stadien kann die Symptomatik jedoch von einer Paratendinitis bzw. -tenonitis überlagert oder begleitet sein. Die Bezeichnung der Tendinitis sollte daher Fällen mit klinisch eindeutig erkennbarem entzündlichem Charakter vorbehalten bleiben (Tab. 2).

Als Ursache einer Tendinopathie werden in erster Linie Mikrorupturen der Kollagenfasern durch lokale Überlastung angenommen. Die Strukturschäden der Sehnenmatrix führen in der Folge zur Aufhebung der hierarchischen Kollagenstruktur. Beschrieben sind Regenerationsprozesse mit einem vermehrtem Aufbau von Kollagen Typ III im Gegensatz zum in gesunden Sehnen vorwiegend zu findenden Kollagen Typ I. Strukturell umgebaute Achillessehnen zeigten in biomechanischen Untersuchungen eine erhöhte Dehnbarkeit bei geringerer Steifigkeit, was bei Spitzendruckbelastungen eine verringerte Reißfestigkeit mit erhöhter Rupturgefahr bedingt [26]. Der ätiologische Ansatz mit lokalisierter Degeneration des Kollagengewebes wurde in histologischen Untersuchungen an mehreren Sehnen (z. B. Achilles- und Patellarsehne, am medialen Ursprung der Plantarfaszie, den Sehnen der Ischiokruralmuskulatur) nachgewiesen $[9,10,27]$. Darüber hinaus wurde in histologischen Untersuchungen an Patienten mit Achillessehnenrupturen auf ein vermehrtes Auftreten von Matrixmetalloproteinasen (MMPs) hingewiesen. Inwiefern diese in der Genese der Tendinopathie eine Rolle spielen, ist allerdings derzeit noch unklar. Ebenso ist bisher nicht geklärt, ob und in welchem Maß einem Impingement durch eine verdickte M.-plantarisSehne in der Entstehung einer Tendinopathie eine bedeutsame Rolle zukommt [28].

Im Rahmen des degenerativen Umbaus konnte ein vermehrtes Vorkommen von Substanz P und eine gefäßbegleitende Einsprossung Substanz P positiver Nervenendigungen gezeigt werden. Die Einsprossung der Nervenendigungen liefert ein Erklärungsmodell für den schmerzhaften Verlauf der Symptomatik. Substanz P spielt ebenso eine entscheidende Rolle in der Entwicklung der Sehnendegeneration. Im Tiermodell konnten eine Steigerung der Tenozytenzahl und Stimulation der Neoangionese nach exogen zugeführter Substanz $P$ nachgewiesen werden, zudem wurde die Entwicklung 
Tab. 1 Mögliche Einflussfaktoren zur Entwicklung von Tendinopathien der unteren Extremität.

\begin{tabular}{|c|c|c|}
\hline Einflussfaktor & Differenzierung & Bewertung \\
\hline $\begin{array}{l}\text { Achsabweichungen } \\
\text { des Skeletts* }\end{array}$ & $\begin{array}{l}\text { z. B. Beinlängendifferenz, erniedrigtes } \\
\text { Fußlängsgewölbe }\end{array}$ & +0 \\
\hline Athletenalter* & & $+o$ \\
\hline \multirow[t]{2}{*}{ Belastungsdosierung** } & Trainingsumfang & ++ \\
\hline & Intensität & +0 \\
\hline $\begin{array}{l}\text { Material und Trainings- } \\
\text { umgebung** }\end{array}$ & & oo \\
\hline $\begin{array}{l}\text { muskuläre Flexibilität } \\
\text { (Dehnfähigkeit)* }\end{array}$ & Quadrizeps- und Hamstring-Muskulatur & $+o$ \\
\hline
\end{tabular}

\begin{tabular}{|c|c|c|}
\hline Schuhwerk** & & oo \\
\hline \multirow[t]{3}{*}{ Sportart** } & Läufer: Achillessehne, Tractus iliotibialis & ++ \\
\hline & Sprungsportarten: Patellarsehne & ++ \\
\hline & Fußball: Sehnen der Adduktorenmuskulatur & ++ \\
\hline Trainingsalter* & & ++ \\
\hline Untergrund** & & oo \\
\hline Vorverletzung* & & ++ \\
\hline \multirow{2}{*}{$\begin{array}{l}\text { (neuro-)muskuläre } \\
\text { Insuffizienz* }\end{array}$} & Kraft der Quadrizepsmuskulatur & +0 \\
\hline & vertikale Sprungleistungsfähigkeit & +0 \\
\hline Trainingsfehler** & Sprungtechnik & +0 \\
\hline \multirow[t]{4}{*}{ Anthropometrie* } & Gewicht & +0 \\
\hline & Größe & +0 \\
\hline & BMI & +0 \\
\hline & Taille-Hüft-Verhältnis & +0 \\
\hline
\end{tabular}

* intrinsisch, ${ }^{* *}$ extrinsisch, ++ durch wissenschaftliche Untersuchungen belegt, +o hohe Praxisrelevanz, wissenschaftlich nicht abschließend und für alle Sportarten geklärt, oo Praxisrelevanz

einer Paratenonitis beobachtet. Substanz $P$ ist in niedriger Konzentration in asymptomatischen Sehnen und vermehrt bei Tendinopathien nachweisbar. Eine artifizielle Reduktion von Substanz $\mathrm{P}$ wird deshalb derzeit als möglicher $\mathrm{Zu}-$ gang in der Therapie von Tendinopathien diskutiert, ist allerdings bisher nicht belegt $[30,31]$.

Diese beschriebenen Strukturveränderungen sind in der bildgebenden Diagnostik (Sonografie, MRT) erst bei längerem Bestehen der Symptomatik nachweisbar. Klinisch manifestiert sich der Umbau zumeist zunächst in einer geringen Dickenzunahme der Sehne. Somit ist die Abgrenzung zur physiologischen Adaptation der Struktur bei sportartspezifisch hohen Impact-Kräften, zumindest am Anfang, schwierig. Als weitere Zeichen des Degenerationsprozesses werden (doppler-)sonografisch visualisierte Echoinhomogenitäten und Neovaskula- risationen gewertet (Abb. 1). Allerdings ist derzeit nicht abschließend geklärt, in welchem Maß die Sehnenvaskularisierung eine physiologische Anpassung auf eine Mehrbelastung darstellt oder als pathologische Adaptation zu werten ist. In fortgeschrittenen Stadien manifestieren sich knötchenförmige Verdickungen, Adhäsionen und Verkalkungen, die meist schon im Rahmen der klinischen Untersuchung tastbar sind. Derartige in der klinischen oder bildgebenden Diagnostik auch an asymptomatischen Sehnen auffallende Veränderungen ohne entzündliche Begleitkomponente können mit dem Begriff der Tendinose beschrieben werden (Tab. 2).

\section{Definition}

Tendinopathien sind schmerzhafte Sehnenveränderungen, die mit einer mehr oder minder ausgeprägten Funktionseinschränkung und nicht mit klinischen und histologischen Entzündungszeichen, wie bei der Tendinitis, einhergehen. In akuten Stadien kann die Tendinopathie von einer Paratendinitis bzw. -tenonitis überlagert oder begleitet sein. Bei Auftreten struktureller Sehnenveränderungen in Sonografie oder MRT ohne klinische Symptomatik kann der Terminus der „Tendinose“ verwendet werden.

\section{Zielgerichtete Diagnostik}

Wertigkeit der Sonografie in der Diagnostik von Tendinopathien

Die Sonografie stellt eine schnell verfügbare, verhältnismäßig leicht zu erlernende und überdies kostengünstige Methode zur Beurteilung oberflächlicher Weichteilstrukturen dar. Darüber hinaus ergibt sich die Möglichkeit zur strukturellen Funktionsprüfung in Echtzeit. Einfache Sehnenparameter wie der a.-p. Durchmesser sind mit modernen Sonografiegeräten von geübten Untersuchern valide und mit hoher Reliabilität erfassbar. In Untersuchungen an Achilles- und Patellarsehnen konnten zwischen erfahrenen Untersuchern unabhängig von der Positionierung des Schallkopfs hohe Korrelationen $(0,68-0,99)$ in Intra- und Interobserververgleichen gemessen werden. Untersuchungen mit konventionellem B-Mode-Ultraschall an Patienten mit Tendinopathien zeigten eine moderate bis exzellente Reliabilität in deren Erkennung. Darüber hinaus sind Verlaufskontrollen von Sehnenpathologien mithilfe sonografischer Verfahren einfach, schnell und mit hoher Reproduzierbarkeit der Befunde möglich. Verglichen zur MRT fand sich eine vergleichbare Sensitivität und Spezifität des Ultraschalls in Untersuchung und Bewertung von Tendinopathien der unteren Extremität.

Weiterentwicklungen sonografischer Techniken in den vergangenen Jahren ermöglichen eine tiefergehende Beurteilung der Sehnenmorphologie. Die Sonoelastografie wurde in den 90er-Jahren zur Darstellung der Gewebehärte entwickelt und zunächst in der Tumordiagnostik zur Differenzierung zwischen gutartigem und bösartigem Gewebe angewandt. In jüngerer Zeit beschäftigten sich einige Arbeitsgruppen mit der Anwendung des Verfahrens im Sehnenschall. Erste Ergebnisse zeigen eine verlässliche Einschätzung in der Beurteilung des Gewebes von Achilles- und Patellarsehnentendinosen [14,32]. 


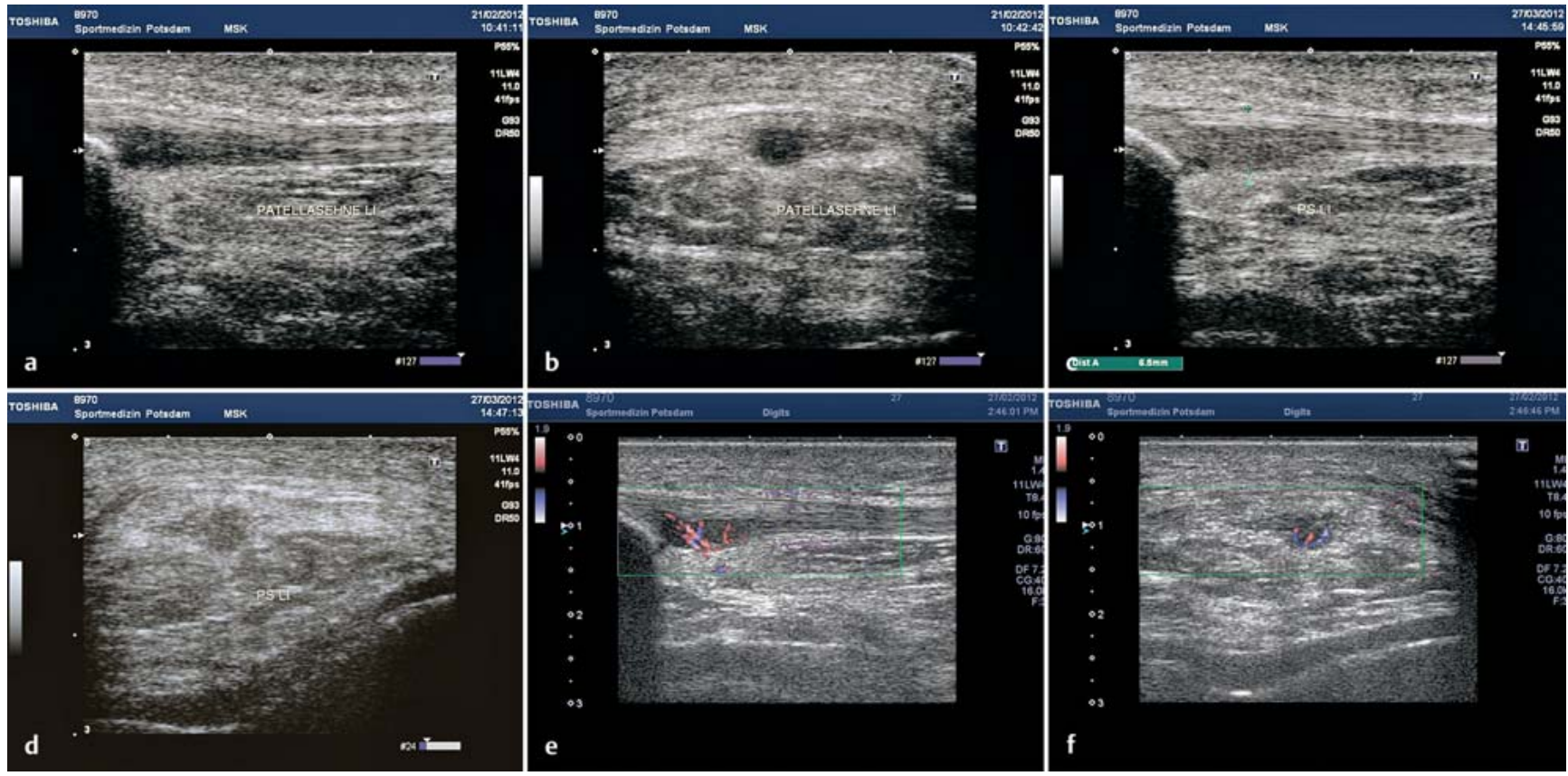

Abb. 1 a, b Ursprungsnahe Tendinopathie der Patellarsehne eines Kaderathleten in Longitudinal- und Transversalschnitt mit einer lokalen Auftreibung im mittleren Sehnendrittel auf ca. $7 \mathrm{~mm}$ und einem Ausmaß der Hypoechogenität von $4 \times 5 \mathrm{~mm}$ transversal gemessen. c, d Befund nach 5-wöchiger Intervention mit exzentrischem Training sowie Aussetzen schmerzauslösender (sportartspezifischer) Belastungen, wie intensive Sprintund Sprungbelastungen. e, $\mathbf{f}$ In der hochauflösenden Power-Doppler-Sonografie („advanced dynamic flow“-Modus, Toshiba) kommt die vaskuläre Aktivität der Neovaskularisationen im Bereich der Hypoechogenität in Longitudinal- und Transversalschnitt zur Darstellung.

Tab. 2 Klassifikation von Sehnenbeschwerden bzw. -pathologien in Anlehnung an [26] und [28].

\begin{tabular}{|c|c|c|c|}
\hline Diagnose & klinischer und sonografischer Befund & histologischer Befund & klinische Zeichen und Symptome \\
\hline Tendinopathie & $\begin{array}{l}\text { diffuser oder lokalisierter Sehnendruck- } \\
\text { schmerz, evtl. Sehnenverdickung sono- } \\
\text { grafisch Dickenzunahme, Hypo- und } \\
\text { Hyperechogenitäten, Neovaskularisierung } \\
\text { möglich }\end{array}$ & $\begin{array}{l}\text { alle Stadien von organisierter, hierar- } \\
\text { chisch geordneter Kollagenstruktur } \\
\text { bis zum Bild der Tendiose (s.u.) }\end{array}$ & $\begin{array}{l}\text { subakuter Beginn mit belastungs- } \\
\text { abhängigen Beschwerden Ein- } \\
\text { schränkung abhängig vom Stadium } \\
\text { und einer möglichen Begleitreak- } \\
\text { tion des Paratenons }\end{array}$ \\
\hline Tendinose & $\begin{array}{l}\text { intratendinöse Degeneration mit Auf- } \\
\text { treibung der Sehne und Adhäsionen } \\
\text { sonografisch messbare Dickenzunahme, } \\
\text { Abbrüche der Kollagenfaserstruktur evtl. } \\
\text { Neovaskularisierung sowie Hypo- und } \\
\text { Hyperechogenitäten }\end{array}$ & $\begin{array}{l}\text { Sehnendegeneration mit Disorganisa- } \\
\text { tion der Kollagenfasern, vermehrter } \\
\text { mukoider Grundsubstanz, Hyperzellu- } \\
\text { larität, Einsprossen kleiner Gefäßen } \\
\text { und Nerven, lokaler Nekrose oder Kal- } \\
\text { zifizierung }\end{array}$ & $\begin{array}{l}\text { in akuten Stadien wie bei Tendino- } \\
\text { pathie, ansonsten subklinischer } \\
\text { bzw. asymtomatischer Verlauf }\end{array}$ \\
\hline Tendinitis & $\begin{array}{l}\text { klinische Zeichen: Schwellung, Rötung, } \\
\text { Überwärmung, Druckschmerz sonogra- } \\
\text { fische Zeichen: diffuse Aufquellung der } \\
\text { Sehne mit hypoechogenen Zonen und } \\
\text { Verlust der hierarchischen Kollagenfaser- } \\
\text { struktur, ausgeprägte Neovaskularisierung }\end{array}$ & $\begin{array}{l}\text { symptomatische Degeneration der } \\
\text { Sehne mit inflammatorischer Repara- } \\
\text { tion wie bei Tendinose: } \\
\text { Faserabbrüche,(myo-)fibroblastische } \\
\text { Proliferation, } \\
\text { Granulationsgewebebildung }\end{array}$ & $\begin{array}{l}\text { akute Schwellung und Schmerzen } \\
\text { mit deutlicher Einschränkung im } \\
\text { Alltag diffuser Sehnendruck- } \\
\text { schmerz deutliche Einschränkung } \\
\text { bei sämtlichen Belastungen }\end{array}$ \\
\hline $\begin{array}{l}\text { Paratenonitis/ } \\
\text { Peritendinitis }\end{array}$ & $\begin{array}{l}\text { „Inflammation“ des Sehnengleitgewebes, } \\
\text { prall-elastische Schwellung, Rötung und } \\
\text { Überwärmung möglich, tastbares „Rei- } \\
\text { ben“ der Sehne hypoechogener Rand- } \\
\text { saum in der Sonografie }\end{array}$ & $\begin{array}{l}\text { Wassereinlagerung in den paratenoni- } \\
\text { tischen/peritendinischen Raum Infil- } \\
\text { tration von Monozyten }\end{array}$ & $\begin{array}{l}\text { akute, prall-elastische Schwellung } \\
\text { Rötung und Überwärmung möglich } \\
\text { akute belastungsabhängige Be- } \\
\text { schwerden }\end{array}$ \\
\hline
\end{tabular}

Andere Verfahren ermöglichen die Quantifizierung der Graustufen bzw. Schallmuster des B-Bildes und verhelfen somit zur Darstellung kleinster Strukturveränderungen im Gewebe. Sie wurden zunächst an gesunden und tendinopathisch veränderten Achillessehnen evaluiert. Die ultrasonografische Gewebecharakterisierung (UTC) untersucht das Gewebe nach dessen dreidimensionaler Rekonstruktion auf die Stabilität der Echosignale bzw. die Häufigkeit von Strangabbrüchen im Gewebe (van Schie 2009). Die Quantifizierung des Sehnen- gewebes mittels räumlicher Frequenzparameter (Spatial Frequency Parameters, Bashford 2008) ermittelt die Graustufenanteile benachbarter Regionen und deren Echofrequenzmuster auf Basis des longitudinalen B-Bildes. Vereinfacht 


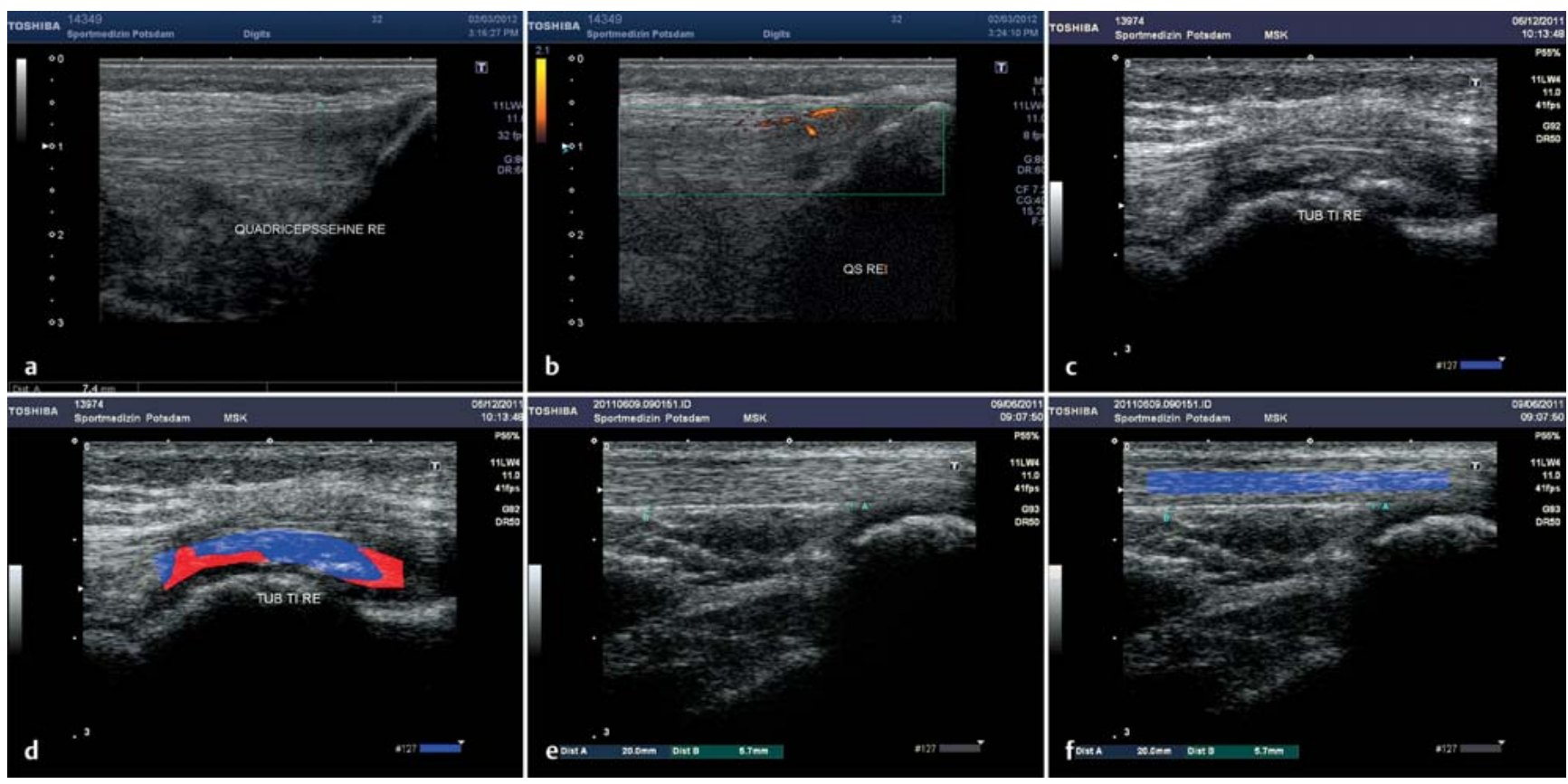

Abb. 2 a, b Tendinopathie der ansatznahen Quadrizepssehne eines 32-jährigen Hobbysportlers (Basketball, Badminton) im B-Bild und in der Power-Doppler-Sonografie. Die Sehne ist im Seitenvergleich um 1,5 mm verdickt und beinhaltet ein längliches hypoechogenes Areal mit $10 \times 2 \mathrm{~mm}$ Ausmaß. c, d Ansatznahe Tendinopathie eines 11-jährigen Nachwuchsathleten bei initialem Morbus Schlatter im longitudinalen B-Bild und nach Quantifizierung der Sehnenstruktur (Spatial Frequency Parameters, [2]. Bereiche blauer Farbe symbolisieren überwiegend unverändertes, solche mit roter Farbe überwiegend verändertes Gewebe. e, f Sonografisch unauffälliger Befund einer Achillessehne eines Nachwuchsathleten im Longitudinalschnitt mit unauffälliger Darstellung in der Quantifizierung mittels räumlicher Frequenzparameter (Spatial Frequency Parameters, [2]). a Exzentrisches Training der Achillessehne (Alfredson). b Nordic Hamstrings. c Sensomotorisches Training unter körpereigener Last der hinteren Kette. d Sensomotorisches Training unter körpereigener Last der unteren Extremität.

kann auf diesem Weg der „Graustufengehalt" der Sehne ermittelt werden.

Ziel ist die möglichst frühe Erkennung von verändertem Kollagengehalt der Sehne als Maß eines Gewebeumbaus (Abb. 2c, d; Vergleich zur gesunden Sehne in Abb. 2 e,f). Beiden Verfahren liegen physikalische Algorithmen zugrunde, die standardisierte Geräteeinstellungen (bezüglich Schallfrequenz, -fokus, -tiefe und -verstärkung) der Sehnenuntersuchung zur Vergleichbarkeit der Graustufenwerte unterschiedlicher Bilder voraussetzen. Mithilfe der Quantifizierung der Graustufen des B-Bildes sollen kleinste Strukturveränderungen im Gewebe darstellbar gemacht werden. Ein bereits etabliertes Verfahren im Sehnenschall zur Beurteilung der Vaskularisierung, die Doppler-Sonografie, wurde vorwiegend an Sehnenpathologien von Achilles- und Patellarsehne sowie der Epikondylitiden untersucht. Breiten Einsatz findet das Verfahren ebenso zur Beurteilung von entzündlichen Aktivitäten von Synovialis und Peritendineum in der rheumatologischen Diagnostik. Hierbei werden sowohl Color- als auch Power-Doppler-Sonografie genutzt, wobei den auf den PowerDoppler-Verfahren basierenden Metho- den eine höhere Sensitivität zur Darstellung kleinster Gefäßflüsse zukommt.

In verschiedenen Arbeiten konnte die reliable Darstellung von Neovaskularisation zwischen unterschiedlichen Untersuchern gezeigt werden [15]. Nach Injektion eines sich in Sehnen anreichernden, farbverstärkenden Kontrastmittels kann eine nochmals verbesserte Darstellung der intratendinösen Gefäße erreicht werden (CEUS: contrast-enhanced ultrasound [33]. Da bislang jedoch nicht endgültig geklärt werden konnte, welche klinische Bedeutung Auftreten und Ausmaß der Neovaskularisationen beizumessen ist bzw. welche Behandlungsstrategie daraus abgeleitet werden muss, ist der Nutzen der frühzeitigen Detektion einzelner Gefäße mithilfe sensitiver Methoden noch fraglich.

Die dopplersonografischen Verfahren werden neben dem Einsatz in der Diagnostik auch in der Behandlung von Tendinopathien eingesetzt. Dopplersonografisch gestützt werden beispielsweise Injektionstherapien mit Polidocanol zur Sklerosierung der Neovaskularisationen angewandt. Darüber hinaus wurden aktuell minimalinvasive Operationstechniken unter dopplersonografischer Kon- trolle vorgestellt, die auf die chirurgische Durchtrennung der Neovaskularisierung mit den die Gefäße begleitenden Nervenästen abzielen [28].

\section{Diagnostik}

Klinik

Am Anfang der Untersuchung steht eine ausführliche Anamnese, die beschwerdeauslösende Situationen während Sport- und Alltagsaktivitäten mit einschließt. Fokussiert werden sollte insbesondere auf die Schmerzentwicklung sowie auf Verlauf und Charakter der Beschwerden. Zu Beginn werden von den Patienten in der Regel Anlaufschmerzen am Morgen nach intensiven Belastungen tags zuvor sowie am Anfang von Belastungen (z.B. Treppengehen oder Sprünge) angegeben. Oft mildern sich die Schmerzen im Tagesverlauf bzw. unter Fortführung der Belastung ab. In der Mehrzahl der Fälle nehmen die Beschwerden bei höheren Intensitäten, insbesondere nach längerer Belastungsdauer, wieder zu.

In der klinischen Untersuchung zeigt sich die Sehne druckschmerzhaft und zumeist mit erhöhtem Spannungszustand 
(höhere Konsistenz) im Seitenvergleich im schmerzhaften Areal. In isometrischen und exzentrischen Funktionsund Belastungstests der zugehörigen Muskulatur lassen sich die Beschwerden reproduzieren [3]. In fortgeschrittenen Stadien sind zudem lokale knotenförmige Auftreibungen der Sehne sicht- bzw. tastbar.

\section{Bildgebende Verfahren}

Je nach Lokalisation sind folglich zusätzliche bildgebende Untersuchungen zur Bestimmung des Ausmaßes der Veränderungen sinnvoll und notwendig. Als erste Wahl ist bei den meisten oberflächlich liegenden Sehnen die sonografische Untersuchung empfehlenswert. Sie ermöglicht die Visualisierung von intratendinösen Läsionen (hypo- und hyperechogene Areale) und Verdickungen (s. Box „Zielgerichtete Diagnostik“).

Bei Ursprungs- bzw. Ansatztendinopathien sollten Veränderungen der angrenzenden Knochenstruktur (z.B. Fersensporn) mittels Nativröntgen ausgeschlossen werden.

Bei länger bestehenden belastungsabhängigen Beschwerden am Ursprung der Beinbeugemuskulatur am Becken ist zur Diagnosesicherung einer Tendinopathie aufgrund der tiefen Lage der Strukturen die MRT-Diagnostik zu erwägen. In einer aktuellen Arbeit wurden bei Patienten mit Beschwerden am Ursprung der Ischiokruralmuskulatur signifikant dickere Sehnen im a.-p.Schnitt gemessen. Charakteristisch war - analog den Befunden von Tendinopathien anderer Lokalisationen - die unabhängig von der Symptomatik bestehende verstärkte T1- und T2-Anreicherung im Peritendineum [4].

Die Diagnose einer Tendinopathie ohne Begleitentzündung im Sport ergibt sich somit abschließend aus folgenden differenzialdiagnostischen Verfahren:

- ausführliche Anamnese inklusive Trainingsanamnese (initiale Beschwerden mit meist Schmerzreduktion bei mäßiger Belastung),

- Lokalbefund (Druckschmerz, Knötchenbildung oder Strukturunregelmäßigkeiten),

- positive Funktionstests (Schmerzangabe bei Belastung),

- apparative Diagnostik (Sonografie, ggf. MRT oder Röntgen).
In der Diagnostik der Tendinopathie spielt neben einer differenzierten klinischen Untersuchung von betroffener Sehne und angrenzender knöcherner Strukturen die ausführliche Schmerzund Belastungsanamnese eine wegweisende Rolle. Bildgebende Verfahren erlauben die valide Beurteilung struktureller Schäden und sollten sowohl in der Erstdiagnostik als auch in Verlaufskontrollen einsetzt werden.

\section{Therapie}

\section{Konservative Behandlung}

Trainingstherapie

Als Mittel der Wahl in der Therapie von Tendinopathien sind trainingstherapeutische Maßnahmen anzusehen. Verschiedene Reviews und Metaanalysen sehen eine hohe Evidenz insbesondere für trainingstherapeutische Programme, die einen hohen Anteil an exzentrischen Sehnenbelastungen beinhalten $[8,11]$. Therapieerfolge mit deutlich verbesserter Schmerzsymptomatik und Funktion konnten v.a. bezüglich der Achillessehnentendinopathie, meist über eine Behandlungsdauer von 12 Wochen, nachgewiesen werden [34]. Klinisch messbare Erfolge lassen sich aber auch bereits nach 4 Wochen erreichen [12] (Abb. 3a). Sonografische Nachuntersuchungen nach exzentrischem Training (im Mittel erfolgte die Nachuntersuchung der Patienten nach etwa 4 Jahren) zeigen eine Normalisierung der Sehnenstruktur und einen Rückgang der Sehnendicke [13]. Unklar ist bislang jedoch, ob die Verbesserung von Schmerz und Funktion auf den Umbau der Sehnenstruktur während der Therapie oder eine optimierte Lastkompensation zurückzuführen ist.

Bevorzugt wurde die Wirkung des exzentrischen Trainings bei Tendinopathien von Achilles- und Patellarsehnen untersucht. Zudem belegen erste Untersuchungen an Patienten mit Tendinopathien der Ischiokruralmuskulatur diese Effekte. Dies legt den Schluss nahe, dass exzentrische Trainingsprogramme bei Tendinopathien auf unterschiedliche Lokalisationen übertragbar und empfehlenswert sind. Der wissenschaftliche Nachweis diesbezüglich ist bislang allerdings nicht für alle Lokalisationen geführt. Exemplarisch wird in Abb. 3 ein multimodales Konzept für die Wadenund die Ischiokruralmuskulatur bzw. die entsprechenden Sehnen dargestellt.

\section{Tipp für die Praxis}

Trainingsdosierung von sensomotorischem und exzentrischem Training

In verschiedenen Studien werden unterschiedliche Möglichkeiten von Häufigkeit, Ausführung und Dosierung der Trainingsprogramme angewendet bzw. vorgeschlagen. Zusammengefasst ist eine 3-5-mal wöchentliche Durchführung der Übungen als Mindestmaß einer Erfolg versprechenden Therapie anzusehen. Angaben zur Ausführung der Trainingsformen bzw. Übungen und der Dosierung (Intensität, Umfang und Dauer der Belastung) differieren in den Studien. Häufig wird davon ausgegangen, die Programme analog eines Hypertrophietrainings (ca. 70-85\% des 1. Wiederholungsmaximums für die jeweilige Belastung) bei hoher Intensität zu absolvieren. Für gut trainierte Sportler und Freizeitsportler ist ein 3-Satz-Training mit bis zu 12 Wiederholungen und einer Pausenlänge zwischen 1 und 3 min zwischen den Sätzen empfehlenswert [35].

Während und nach exzentrischen Belastungen sind Schmerzen im Bereich der behandelten Sehne zu erwarten. Bei zunehmenden Schmerzen während der Belastung ist ein Abbruch der Übung in der Regel nicht notwendig, sofern der Schmerz nach der Belastung wieder nachlässt. Andernfalls ist die Anpassung der Intensität notwendig. Eine Schmerzquantifizierung, beispielsweise mithilfe einer visuellen Analogskala, erlaubt Therapeut und Arzt eine valide Verlaufsbeurteilung. Eine Steigerung der Intensität (Last/Erhöhung des Schwierigkeitsgrads) wird von einigen Autoren bereits bei rückläufigem Schmerz während der Übungsausführung empfohlen. Als oberstes Ziel sollte jedoch die möglichst hohe Bewegungsqualität bei der Übungsausführung gelten.

Individuelle Heimprogramme ermöglichen die eigenständige Fortführung der Trainingsintervention mit schrittweiser Steigerung der Belastung. Der Trainingsplan sollte neben der Intensitätssteigerung auch eine Erhöhung des Umfangs beinhalten. Bestenfalls sollte in regelmäßigen Abständen (zunächst wöchentlich, danach $1 / 4$ - bis $1 / 2$-jährlich) eine Überprüfung bzw. Anpassung von Ausführungsqualität bzw. Belastungsintensität durch den Therapeuten erfolgen. Auf diesem Weg wird ebenfalls eine Compliance-Kontrolle ermöglicht, die für den langfristigen Therapieerfolg von großer Bedeutung ist. 

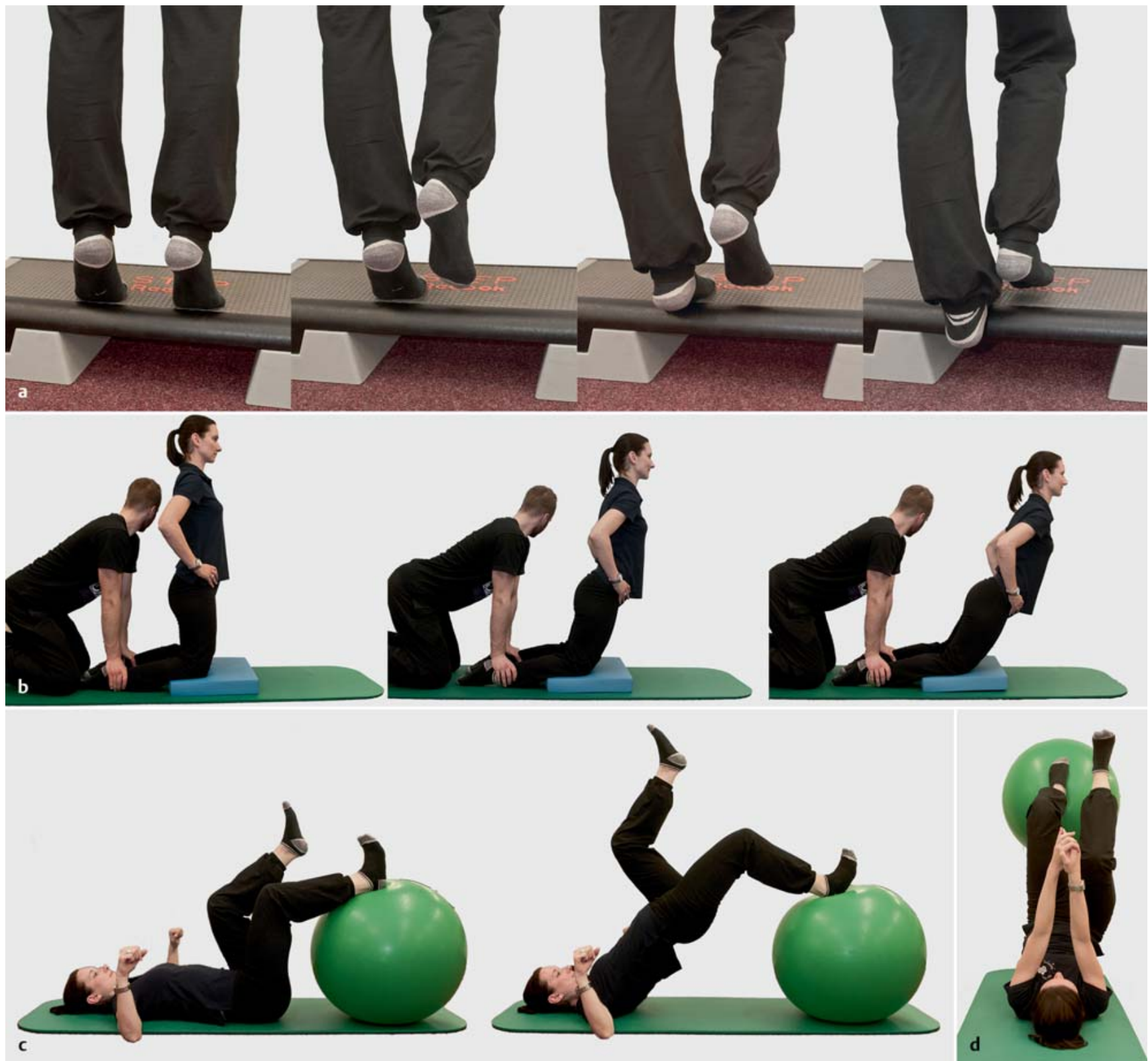

Abb. 3 Exzentrisches und sensomotorisches Training (multimodales Trainingsprogramm) zur Behandlung von Tendinopathien ausgewählter Lokalisationen an der unteren Extremität. a Exzentrisches Training der Achillessehne (Alfredson). b Nordic Hamstrings. c Sensomotorisches Training unter körpereigener Last der hinteren Kette. d Sensomotorisches Training unter körpereigener Last der unteren Extremität.

Beachtenswert bei der Interpretation der Studienergebnisse ist, dass die genannten Trainingsprogramme in der Regel mehrmals wöchentlich bis täglich absolviert wurden. Eine 3-5-mal wöchentliche Anwendung wird daher derzeit als Mindestmaß einer Erfolg versprechenden Therapie angesehen. Angaben zur Art und Weise der Ausführung der Trainingsformen bzw. deren exakte Durchführung und Dosierung (Intensität, Umfang und Dauer der Belastung) schwanken je nach Autor erheblich (s. „Tipp für die Praxis“). Bestenfalls kann eine Anleitung der Übungen durch einen Sport- oder Physiotherapeuten erfolgen. Spätestens nach einigen Wochen The- rapiedauer sollten die Patienten mit individuellen Heimprogrammen zur Umfangserhöhung ausgestattet werden (Abb.4). Diese ermöglichen die eigenständige Fortführung der Trainingsintervention mit schrittweiser Steigerung der Belastung. In regelmäßigen Abständen ist eine Überprüfung bzw. Anpassung von Ausführungsqualität bzw. Belastungsintensität durch den Therapeuten sinnvoll. Auf diese Weise wird zusätzlich eine Compliance-Kontrolle ermöglicht, die für die weitere therapeutische Vorgehensweise von großer Bedeutung ist. Durch regelmäßige Rücksprachen zwischen Therapeut und Arzt sowie Wiedervorstellungen des Patienten in der ärztlichen Sprechstunde wird die Belastungsintensität an die aktuelle Belastbarkeit und den Verlauf der strukturellen und klinischen Adaptation angepasst. Die enge und intensive Zusammenarbeit zwischen Therapeut und Arzt ist dabei von grundlegender Bedeutung für eine erfolgreiche Therapie.

\section{Stoßwellentherapie}

Die Stoßwellentherapie (SWT) zeigte bisweilen bei Tendinopathiepatienten unterschiedlicher Regionen positive Ergebnisse auf Schmerzreduktion und Funktionsverbesserung. In Literaturübersichten wird die extrakorporale 


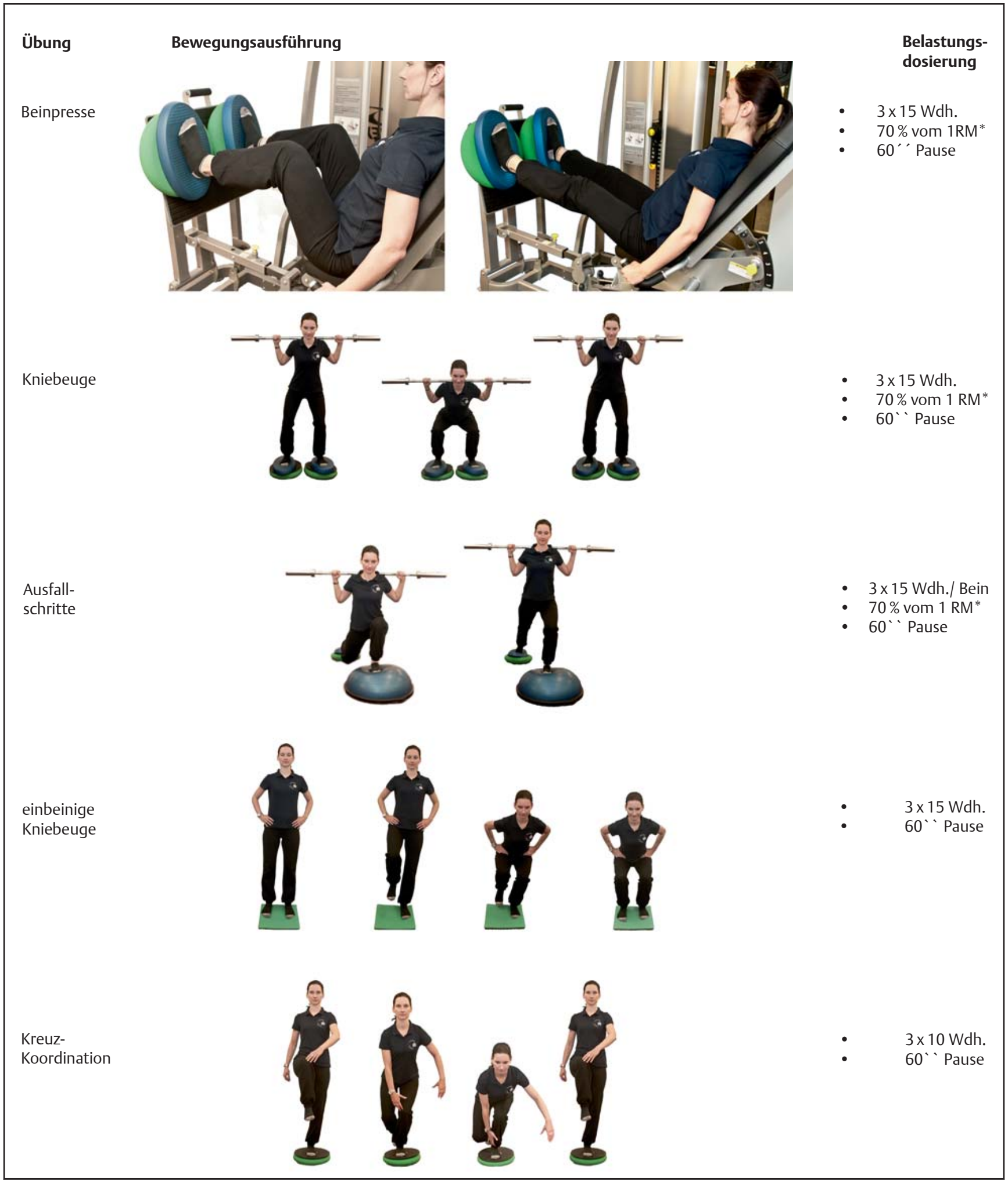

Abb.4 Geführtes sowie freies sensomotorisches und exzentrisches Training in der Sporttherapie am Beispiel der Patellarsehnentendinopathie. Je nach gewünschtem Schwierigkeitsgrad können unterschiedliche instabile Unterlagen verwendet und ggf. auch kombiniert werden. Eine Belastungssteigerung kann im Heimprogramm mit Erhöhung von Last und/oder Schwierigkeitsgrad durch Wechsel der Unterlage erfolgen. ( ${ }^{*}$ Das 1 RM ist das maximale Gewicht, mit dem eine Übung einmalig bei sauberer Technik ausführbar ist). 
Stoßwellentherapie (ESWT) besonders bei Ansatztendinopathien der Achillessehne favorisiert, bei denen exzentrische Trainingsmaßnahmen weniger gute Ergebnisse zeigen $[9,11]$. Bezüglich der Patellarsehnentendinopathie wurde die Effektivität hingegen als begrenzt eingestuft [8]. In der sog. TOPSHOCK-Studie werden derzeit die Wirkungen der ESWT im Vergleich zur radialen SWT bei der Patellarsehnentendinopathie überprüft [18]. Die Ergebnisse dieser Untersuchungen werden die zukünftigen Therapiestrategien möglicherweise beeinflussen. Nicht selten wird die SWT im Sinne einer multimodalen Therapie mit exzentrischem Training kombiniert $[11,36]$.

\section{Physiotherapeutische Verfahren}

Obwohl in der Praxis vielfach angewendet ist die Evidenz für die unterschiedlichen Therapieformen in der Physiotherapie wie Querfriktionen, Ultraschalltherapie, Elektrotherapie, Kryotherapie oder Stretching bezüglich sämtlicher Lokalisationen der unteren Extremität nicht abschließend geklärt. Ziele dieser Techniken sind einerseits die Schmerzreduktion und andererseits mechanische Effekte auf struktureller Ebene. So wird beispielsweise Querfriktionen neben dem Lösen von Adhäsionen eine vermehrte Stimulation der Fibroblasten mit in der Folge erhöhter Kollagennettosynthese zugeschrieben. Ähnliche Mechanismen werden für die Ultraschalltherapie diskutiert. In einer aktuellen Literaturübersicht zur Patellarsehnentendinopathie wurde die Ultraschalltherapie allerdings als unwirksam betrachtet. Dagegen konnte in einer kombinierten Behandlung der Achillessehnentendinopathie mit Querfriktionen, Ultraschall, exzentrischem und sensomotorischem Training eine Besserung von Funktion und Schmerz der Patienten gezeigt werden.

Nicht steroidale Antirheumatika (NSAR)

Die Indikation zur Einnahme von NSAR bei chronischen, belastungsabhängigen Sehnenbeschwerden wird bereits seit Längerem diskutiert. Trotz fehlendem Nachweis von Entzündungszellen und -mediatoren im tendinopathischen Sehnengewebe und einer nicht nachgewiesenen Evidenz ist der Einsatz insbesondere von Diclofenac und Ibuprofen weiterhin weit verbreitet. Bei Auftreten eines akuten peritendinitischen Begleitprozesses erscheint der Einsatz dieser
Medikamente hingegen gerechtfertigt. Darüber hinaus wird diskutiert, dass infolge der schmerzreduzierenden Wirkung der NSAR die Durchführung von physio- und trainingstherapeutischen Maßnahmen erleichtert werden kann. Dies trifft insbesondere auf Trainingsformen zu, die zur Behandlung schmerzbedingt veränderter Bewegungsmuster appliziert werden. Auf diese Weise könnte eine zügigere Rehabilitation von Patienten und Athleten begünstigt werden. Die Indikation zur Verordnung von NSAR muss daher im individuellen Fall erwogen werden und sollte zeitlich begrenzt (max. 1-2 Wochen) sein, mit dem Ziel der Unterstützung des vorgesehenen therapeutischen Konzepts sein.

\section{Injektionsbehandlungen}

\section{Polidocanol}

Unter der Annahme, dass im tendinopathischen Gewebe nachgewiesene Neovaskularisationen und Schmerzfasern die Symptomatik der Pathologie unterhalten, wurden bevorzugt im skandinavischen Raum Sklerosierungsbehandlungen dieser intratendinösen Gefäße unter dopplersonografischer Kontrolle durchgeführt. In zahlreichen Studien bei Achilles- und Patellarsehnentendinopathien sowie Tendinopathien an den Epikondylitiden wurde über signifikante Verbesserungen von Funktion und Schmerz berichtet. Hoksrud u. Mitarb. fanden in ihrer 2-Jahres-Nachuntersuchung nach 5-maliger Polidocanolanwendung bei 120 Patienten mit Patellarsehnentendinopathien eine statistisch signifikante Verbesserung des VISA-PScores. Beschwerdefreiheit im Sinne eines Punktewerts über 95 wurde jedoch lediglich bei $20 \%$ der Patienten erreicht. Darüber hinaus sprachen 36\% der Patienten nicht auf die Therapie an (VISA-P $<50$ ). Aufgrund der fehlenden Kontrollgruppe sind die Ergebnisse jedoch mit Zurückhaltung zu interpretieren [7]. In einer aktuellen randomisiert-kontrollierten Studie an 52 Patienten mit Patellarsehnentendinopathie wurde die Verbesserung des Schmerzes durch eine Sklerosierung mit Polidocanol (maximal 3 Injektionen) im Vergleich mit einem arthroskopischen Shaving der Sehnen untersucht. Im Rahmen der ersten Nachuntersuchung zeigten zwar beide Gruppen gute klinische Ergebnisse, bei den Patienten der operativen Behandlung wurde allerdings eine statistisch signifikant bessere Kurzzeitschmerzreduktion (VAS) in Ruhe gemessen [28]. Langzeitergebnisse aus randomisiert-kontrollier- ten Studien, insbesondere im Vergleich mit evidenzbasiert gesichert wirksamen trainingstherapeutischen Behandlungsstrategien, stehen derzeit an sämtlichen Lokalisationen noch aus.

\section{Kortison}

Trotz fehlender pathophysiologischer Begründung bei nicht nachgewiesener Inflammation zeigen Kortisoninjektionen sowohl bei Achillessehnen- als auch bei Patellarsehnentendinopathien Kurzzeiteffekte, die allerdings nicht über den Effekt anderer konservativer Behandlungsalgorithmen hinausgehen. Langzeitergebnisse aus randomisiert-kontrollierten Studien zur Wirkung von Kortison bei chronischen Tendinopathien liegen derzeit nicht vor. In einer Nachuntersuchung zur Wirkung einer ultraschallgesteuerten Kortisoninjektion bei Ursprungstendinopathien der Ischiokruralmuskulatur zeigten sich die Beschwerden nach 6 Monaten bei noch 25\% der Patienten persistent. Belegt sind dagegen unerwünschte Nebenwirkungen der Kortisonbehandlung mit Mikro-, Teil- und Komplettrupturen von Sehnen. Der Einsatz von Kortisoninjektionen sollte daher, zumindest im intratendinösen Verlauf, unterbleiben.

\section{Andere Substanzen}

Vereinzelte Arbeiten berichten über positive Wirkungen bei Anwendung von Injektionen mit Aprotinin, hyperosmolarer Dextrose oder die topische Anwendung von Glycerylnitraten. Die Effektivität dieser Substanzen in der Behandlung von Tendinopathien kann jedoch aufgrund der sehr spärlichen Datenlage derzeit nicht beurteilt werden. Die vielfach propagierte Applikation von Platelet Rich Plasma (PRP) zeigte sich in einer kürzlich veröffentlichten randomisiertkontrollierten Studie als unwirksam. An Patienten mit Achillessehnentendinopathie wurde die Wirkung von PRP mit einer Placeboinjektion mit $\mathrm{NaCl}$ zusätzlich zu exzentrischem Training verglichen. Weder in Bezug auf Schmerz und Funktion noch auf die Gewebemorphologie - ermittelt über die sonografische Gewebecharakterisierung (UTC) sowie das Ausmaß der Neovaskularisierung mittels Doppler-Ultraschall (CDU) zeigte sich zwischen den Gruppen ein Unterschied. Die in beiden Gruppen vorhandenen positiven Therapieeffekte werden von den Autoren auf das von allen Patienten identisch absolvierte exzentrische Trainingsprogramm zurückgeführt [5]. 


\section{Weitere Verfahren}

\section{Sporteinlagen}

Der Einsatz von Schuheinlagen im Sport wird zur Behandlung belastungsbedingter Beschwerden der unteren Extremität zumeist als zusätzliches Verfahren in ein multimodales Therapiekonzept integriert. In neueren, randomisierten, kontrollierten Untersuchungen zeigte sich jedoch, dass auch ein alleiniger Einsatz von Einlagen in der Therapie von Überlastungsbeschwerden der unteren Extremität gute bis sehr gute Effekte auf die Schmerzreduktion (zumindest in Einzelfall) haben kann [6]. Voraussetzung dabei ist allerdings die Verwendung semiindividuell angefertigter Einlagen auf Basis der plantaren Druckverteilung. Als möglicher Wirkmechanismus wird von den Autoren meist die Modulation der Muskelaktivität (beispielsweise des M. peronaeus) diskutiert [37]. Besonders wirksam zeigte sich der Einsatz in der Therapie von Tendinopathien der Achilles- und Patellarsehne sowie dem Tractus-iliotibialis-Syndrom [6].

\section{Operative Behandlung}

Bei fehlendem Ansprechen der Patienten auf konservative Therapieverfahren nach 3-6 Monaten konsequenter Behandlung wird von einigen Autoren die Indikation zum operativen Vorgehen gesehen [1, 38]. In verschiedenen Arbeiten wird über größtenteils gute bis exzellente Ergebnisse bei Tendinopathien unterschiedlicher Lokalisationen berichtet [10]. In erster Linie erfolgt dabei ein Débridement mit Abtragung des tendinopathisch veränderten Gewebes mit Entfernung bzw. Inzision der vom Peritendineum einsprossenden Neovaskularisationen. Allerdings ist bislang der Nutzen des Entfernens der veränderten Gewebeareale für die Patienten nicht abschließend geklärt [38]. Des Weiteren fehlen derzeit standardisierte und evidenzbasierte Nachbehandlungsschemata nach chirurgischem Vorgehen.

In den vergangenen Jahren wurden zunehmend minimalinvasive Operationstechniken zur Behandlung höhergradiger Tendinopathien an unterschiedlichen Lokalisationen entwickelt. Alfredson berichtet über sehr gute Ergebnisse mit deutlicher Schmerzreduktion bei über $90 \%$ der operierten Patienten mittels der sog. „Scraping-Methode“ bei Achillessehnentendinopathien [28]. Unter dopplersonografischer Kontrolle wird am ventralen Peritendineum ent-

Tab. 3 Evidenz zur Wirksamkeit verschiedener Maßnahmen in der Behandlung von Tendinopathien (bevorzugt Achilles- und Patellarsehne).

\begin{tabular}{lll} 
Therapieform & Differenzierung & Bewertung \\
\hline Trainingsformen & exzentrisches Training & ++ \\
\cline { 2 - 3 } & Heavy Slow Resistance Training & ++ \\
\hline Stoßwellentherapie & rensomotorisches Training & +0 \\
\hline \multirow{2}{*}{$\begin{array}{ll}\text { physiotherapeutische } \\
\text { Maßnahmen }\end{array}$} & Quekussierte ESWT & ++ \\
\hline \multirow{2}{*}{ Sporteinlagen } & Ultraschalltherapie & ++ \\
\hline Injektionstherapien & Polidocanol & oo \\
\cline { 2 - 3 } & Kortison & $++/+0$ \\
\cline { 2 - 3 } & Platelet-Rich-Plasma (PRP) & $++/+0$ \\
\hline & $\begin{array}{l}\text { andere Verfahren (z. B. Aprotinin, Dextrose } \\
\text { und Hämolysate) }\end{array}$ & + oo \\
\hline
\end{tabular}

+++ hohe Wirksamkeit, ++ moderate Evidenz, +o geringe bzw. fragliche Wirksamkeit/ggf. Praxisrelevanz, wissenschaftlich nicht abschließend geklärt. oo kein Nachweis bzw. keine Wirkung.

lang der stark vaskularisierten Zone eine Inzision in Längsrichtung durchgeführt. Die Behandlung zielt somit ähnlich der Sklerosierung auf die Unterbrechung der Neovaskularisierung und der parallel verlaufenden Nervenfasern. In einer Nachuntersuchung zeigte sich allerdings nach 18 Monaten kein statistisch signifikanter Unterschied im Vergleich zum perkutanen „Scraping-Verfahren“ mittels Kanüle (VAS-Werte: 69 auf 6; 73 auf 2). Ein zusätzlicher Nutzen durch die offene Behandlung bleibt daher zu diskutieren. Grundsätzlich sind bislang keine prospektiven Daten zum Behandlungsergebnis nach chirurgischer Therapie verfügbar, sodass die Ergebnisse der Studien mit operativer Intervention mit Zurückhaltung interpretiert werden müssen.

\section{Beurteilung}

Patienten mit Tendinopathien verschiedener Lokalisationen sprechen in der Regel gut bis sehr gut auf konservative Therapieverfahren an. Exzentrisches Training zeigt bereits nach wenigen Monaten zum Teil hervorragende Behandlungsergebnisse bei Patienten mit Achilles- und Patellarsehnentendinopathien. Im frühen Stadium der Tendinopathie sind bei vielen Patienten bereits wenige Behandlungen mit exzentrischem Training, ggf. in Kombination mit sensomotorischen und physiotherapeutischen Maßnahmen, ausreichend, um Schmerz- freiheit oder zumindest eine deutliche Schmerzreduktion zu erreichen. Bei höhergradiger Pathologie und länger bestehenden Beschwerden ist hingegen von einem deutlich längeren Therapiezeitraum (in der Regel mindestens 3 Monate) auszugehen. Multimodale Therapieansätze sind dabei meistens sinnvoll. Bei ausgeprägten Befunden und längerer Therapiedauer sind häufigere ärztliche Verlaufskontrollen zur Überprüfung der Belastbarkeit mit nachfolgender Anpassung des Therapieschemas (ggf. in direkter Rücksprache mit dem Therapeuten) nötig.

Erst bei Versagen der konservativen Behandlung über zumindest mehrere Monate sollte der Einsatz eines Operationsverfahrens erwogen werden. Ziel dabei ist vorrangig, den Patienten durch Schmerzlinderung ein intensiviertes Training zu ermöglichen.

Zur Behandlung von Tendinopathien anderer Lokalisationen als an Achilles- und Patellarsehnen liegen bislang nur unzureichende wissenschaftlich überprüfte Daten vor, um eine abschließende Beurteilung evidenter Therapiekonzepte zu erlauben. Meist wird daher das in der Therapie von Achilles- und Patellarsehnentendinopathien generierte Wissen auf andere Lokalisationen übertragen und dort modifiziert angewandt. Inwiefern diese Übertragung gerechtfertigt ist, muss in zukünftigen Längsschnittstu- 
dien mit wissenschaftlichen Designs überprüft werden.

Tab. 3 zeigt eine aktuelle Übersicht der Studienlage zur Evidenz der üblicherweise genutzten Therapieverfahren in der Behandlung von Tendinopathien.

Exzentrisches Training ist in der Therapie von Tendinopathien effektiv und sollte daher derzeit als Behandlungsmaßnahme der ersten Wahl betrachtet werden. Zur Verbesserung der Lastkompensation ist die parallele Durchführung von sensomotorischen Übungen unter Last empfehlenswert. Die Belastungshäufigkeit reicht in Abhängigkeit des Trainingsumfangs von 3-mal wöchentlich bis mehrmals täglich. Dabei scheinen möglichst hohe Intensitäten im Training (an der Schmerzgrenze) am effektivsten. Die Stoßwellentherapie zeigt besonders bei Ursprungs- und Ansatztendinosen gute klinische Ergebnisse. Langzeitergebnisse zur abschließenden Beurteilung der Effektivität bei Tendinopathien stehen allerdings noch aus. Physiotherapeutische Maßnahmen und Sporteinlagen sind allenfalls in Kombination mit den genannten trainingstherapeutischen Maßnahmen sinnvoll. Bei Versagen der konservativen Therapieschemata können unterschiedliche Operationstechniken in Betracht gezogen werden.

Interessenkonflikt: Nein

\section{Literatur}

${ }^{1}$ Amlang MH, Maffuli N, Longo G et al. Operative Behandlung der Achillessehnenruptur. Unfallchirurg 2010; 113: 712-720

2 Bashford GR, Tomsen N, Arya S et al. Tendinopathy Discrimination by Use of Spatial Frequency Parameters in Ultrasound B-Mode Images. IEEE Trans Med Imaging 2008; 27 : 608-615

${ }^{3}$ Cacchio A, Borra F, Severini G et al. Reliability and validity of three pain provocation tests used for the diagnosis of chronic proximal hamstring tendinopathy. Br J Sports Med 2012 [Epub ahead of print]

${ }^{4}$ De Smet AA, Blankenbaker DG, Alsheik NH et al. MRI appearance of the proximal hamstring tendons in patients with and without symptomatic proximal hamstring tendinopathy. Am J Roentgenol 2012; 198: 418-422

${ }^{5}$ de Vos RJ, Weir A, Tol JL et al. No effects of PRP on ultrasonographic tendon structure and neovascularisation in chronic midportion Achilles tendinopathy. Br J Sports Med 2011; 45: 387-392

${ }^{6}$ Hirschmüller A, Baur H, Müller S et al. Clinical effectiveness of customised sport shoe orthoses for overuse injuries in runners: a randomised controlled study. Br J Sports Med 2011; 45: 959-965

${ }^{7}$ Hoksrud A, Torgalsen T, Harstad H et al. Ultrasound-Guided Sclerosis of Neovessels in Patellar Tendinopathy: A Prospective Study of 101 Patients. Am J Sports Med 2012; 40 : 542-547
${ }^{8}$ Larsson ME, Käll I, Nilsson-Helander K. Treatment of patellar tendinopathy - a systematic review of randomized controlled trials. Knee Surg Sports Traumatol Arthrosc 2011 [Epub ahead of print]

${ }^{9}$ League AC. Current concepts review: plantar fasciitis. Foot Ankle Int 2008; 29: 358-366

10 Lempainen L, Sarimo J, Mattila K et al. Proximal hamstring tendinopathy: results of surgical management and histopathologic findings. Am J Sports Med 2009; 37: 727-734

${ }^{11}$ Magnussen RA, Dunn WR, Thomson AB. Nonoperative Treatment of Midportion Achilles Tendinopathy: A Systematic Review. Clin J Sport Med 2009; 19: 54-64

12 Mayer F, Hirschmüller A, Müller S et al. Effects of short-term treatment strategies over 4 weeks in Achilles tendinopathy. Br J Sports Med 2007: 41: e6

13 Ohberg L, Alfredson $H$. Effects on neovascularisation behind the good results with eccentric training in chronic mid-portion Achilles tendinosis? Knee Surg Sports Traumatol Arthrosc 2004; 12: 465-470

14 Rist HJ, Mauch M. Quantifizierte TDI-Elastosonografie der Patellarsehne bei Sportlern. Sportverletz Sportschaden 2012; 26: 27-32

15 Sengkerij PM, de Vos RJ, Weir A et al. Interobserver reliability of neovascularization score using power Doppler ultrasonography in midportion achilles tendinopathy. Am J Sports Med 2009; 37: 1627-1631

16 Tenforde AS, Sayres LC, McCurdy ML et al. Overuse injuries in high school runners: lifetime prevalence and prevention strategies. PM R 2011; 3: 125-131; Quiz 131

17 Cassel M, Baur H, Hirschmüller A et al. Prevalence of Achilles and patellar tendinopathy and their association to intratendinous changes in adolescent athletes. Scand J Med Sci Sports 2014; Sep 11. DOI: 10.1111/ sms.12318 [Epub ahead of print]

18 van der Worp $H$, Zwerver J, van den AkkerScheek I et al. The TOPSHOCK study: effectiveness of radial shockwave therapy compared to focused shockwave therapy for treating patellar tendinopath - design of a randomised controlled trial. BMC Musculoskelet Disord 2011; 12: 229

19 Cook JL, Purdam CR. Rehabilitation of lower limb tendinopathies. Clin Sports Med 2003; 22: 777-789

${ }^{20}$ Hart L. Corticosteroid and other injections in the management of tendinopathies: a review. Clin J Sport Med 2011; 21: 540-541

${ }^{21}$ Hirschmüller A, Frey V, Konstantinidis L et al. Prognostic Value of Achilles Tendon Doppler Sonography in Asymptomatic Runners. Med Sci Sports Exerc 2012; 44: 199-205

22 de Jonge S, de Vos RJ, Weir A et al. One-year follow-up of platelet-rich plasma treatment in chronic Achilles tendinopathy: a doubleblind randomized placebo-controlled trial. Am J Sports Med 2011; 39: 1623-1629

${ }^{23}$ Mayer F, Müller S, Hirschmüller A et al. Die Effizienz konservativer Therapiemaßnahmen bei Tendinopathien im Sport. Dtsch Z Sportmed 2008; 59: 251-254

${ }^{24}$ Hölmich P, Hölmich LR, Bjerg AM. Clinical examination of athletes with groin pain: an intraobserver and interobserver reliability study. Br J Sports Med 2004; 38: 446-451

${ }^{25}$ Hirschmuller A, Frey V, Deibert P et al. Achilles tendon power Doppler sonography in 953 long distance runners - a cross sectional study. Ultraschall Med 2010; 31: 387-393

${ }^{26}$ Arya S, Kulig K. Tendinopathy alters mechanical and material properties of the Achilles tendon. J Appl Physiol 2010; 108: 670-675

27 Maffulli N, Wong J, Almekinders LC. Types and epidemiology of tendinopathy. Clin Sports Med 2003; 22: 675-692
28 Alfredson $H$. Midportion Achilles tendinosis and the plantaris tendon. $\mathrm{Br} \mathrm{J}$ Sports Med 2011; 45: 10233-11025

${ }^{29}$ van Dijk CN, van Sterkenburg MN, Wiegerinck JI et al. Terminology for Achilles tendon related disorders. Knee Surg Sports Traumatol Arthrosc 2011; 19: 835-841

${ }^{30}$ Andersson G, Backman LJ, Scott A et al. Substance $P$ accelerates hypercellularity and angiogenesis in tendon tissue and enhances paratendinitis in response to Achilles tendon overuse in a tendinopathy model. Br J Sports Med 2011; 45: 1017-1022

${ }^{31}$ Tol JL, Spiezia F, Maffulli N. Neovascularization in Achilles tendinopathy: have we been chasing a red herring? Knee Surg Sports Traumatol Arthrosc 2012; 20: 1891-1894

${ }^{32}$ Klauser AS, Faschingbauer R, Jaschke WR. Is sonoelastography of value in assessing tendons? Semin Musculoskelet Radiol 2010; 14: 323-333

33 Genovese E, Ronga M, Recaldini C et al. Analysis of achilles tendon vascularity with second-generation contrast-enhanced ultrasound. J Clin Ultrasound 2011; 39: 141-145

${ }^{34}$ Alfredson H, Cook J. A treatment algorithm for managing Achilles tendinopathy: new treatment options. Br J Sports Med 2007; 41: 211-216

35 Ratamess NA, Alvar BA, Evetoch TK et al. American College of Sports Medicine position stand. Progression models in resistance training for healthy adults. Med Sci Sports Exerc 2009; 41: 687-708

${ }^{36}$ Rompe JD, Nafe D, Furia JP et al. Eccentric loading, shock-wave treatment, or a waitand-see policy for tendinopathy of the main body of tendo Achillis: a randomized controlled trial. Am J Sports Med 2007; 35: $374-$ 383

37 Baur H, Hirschmüller A, Müller S et al. Neuromuscular Activity of the Peroneal Muscle after Foot Orthoses Therapy in Runners. Med Sci Sports Exerc 2011; 43: 1500-1506

38 Maffulli N, Longo UG, Hüfner T et al. Surgical treatment for pain syndromes of the Achilles tendon. Unfallchirurg 2010; 113: 721-725

\section{Institutsangaben}

Michael Cassel, Josefine Stoll, Frank Mayer Hochschulambulanz der Universität Potsdam, Zentrum für Freizeit-, Gesundheits- und Leistungssport

\section{Dr. med. Michael Cassel}

Hochschulambulanz der Universität Potsdam, Professur für Sportmedizin und Sportorthopädie Am Neuen Palais 10, Haus 12 14469 Potsdam

mcassel@uni-potsdam.de

\section{Prof. Dr. med. Frank Mayer}

Ärztlicher Direktor

Hochschulambulanz der Universität Potsdam, Professur für Sportmedizin und Sportorthopädie

Am Neuen Palais 10, Haus 12

14469 Potsdam

fmayer@uni-potsdam.de 